\title{
Lift expectations of random sets
}

\author{
Marc-Arthur Diaye $^{\mathrm{a}}$, Gleb A. Koshevoy ${ }^{\mathrm{b}}$, Ilya Molchanov ${ }^{\mathrm{c}, *}$ \\ ${ }^{a}$ CES, University Paris 1 Pantheon-Sorbonne, France \\ ${ }^{b}$ The Institute for Information Transmission Problems, Bolshoy Karetny 19, 127051 \\ Moscow, Russia \\ ${ }^{c}$ University of Bern, Institute of Mathematical Statistics and Actuarial Science, \\ Alpeneggstrasse 22, 3012 Bern, Switzerland
}

\begin{abstract}
It is known that the distribution of an integrable random vector $\xi$ in $\mathbb{R}^{d}$ is uniquely determined by a $(d+1)$-dimensional convex body called the lift zonoid of $\xi$. This concept is generalised to define the lift expectation of random convex bodies. However, the unique identification property of distributions is lost; it is shown that the lift expectation uniquely identifies only one-dimensional distributions of the support function, and so different random convex bodies may share the same lift expectation. The extent of this nonuniqueness is analysed and it is related to the identification of random convex functions using only their onedimensional marginals. Applications to construction of depth-trimmed regions and partial ordering of random convex bodies are also mentioned.
\end{abstract}

Keywords: random set, selection expectation, lift zonoid, support function, risk measure, outlier

2010 MSC: 62H11, 60D05

\section{Introduction}

Probability theory provides numerous ways of identifying distributions of random variables. Mentioning two (rather nontraditional) examples, the distribution of an integrable random variable $\xi$ is uniquely determined by its stop-loss transform $\mathbf{E}(t+\xi)_{+}, t \in \mathbb{R}$, where $x_{+}$denotes the positive part of $x \in \mathbb{R}$. Furthermore, Hoeffding (1953) showed that the sequence $\mathbf{E} \max \left(\xi_{1}, \ldots, \xi_{n}\right), n \geq 1$, built from i.i.d. copies of $\xi$ uniquely determines the distribution of $\xi$.

Extensions of these identification results to random vectors are of a geometric nature and rely on the concept of zonoids and zonotopes. Zonotopes form an important family of polytopes, which are defined as Minkowski (elementwise) sums of a finite number of segments. Zonoids are convex sets that appear as

\footnotetext{
* Corresponding author

Email addresses: marc-arthur.diaye@univ-paris1.fr (Marc-Arthur Diaye), koshevoy@cemi.rssi.ru (Gleb A. Koshevoy), ilya.molchanov@stat.unibe.ch (Ilya Molchanov)
}

Preprint submitted to Statistics and Probability Letters

July 5, 2021 
limits in the Hausdorff metric of a sequence of zonotopes, see (Schneider, 2014, Sec. 3.5). In the plane, all (centrally) symmetric convex sets are zonoids, while the symmetry is only a strictly necessary condition in dimensions three and more.

Zonoids can also be described as expectations of random segments. For this purpose, recall that a random convex closed set $X$ in $\mathbb{R}^{d}$ is a map from a probability space $(\Omega, \mathfrak{F}, \mathbf{P})$ to the family of convex closed sets in $\mathbb{R}^{d}$, which is measurable in the sense that $\{\omega: X(\omega) \cap K \neq \emptyset\} \in \mathfrak{F}$ for all compact sets $K$ in $\mathbb{R}^{d}$, see (Molchanov, 2017, Def. 1.1.1). If $X$ is almost surely compact and non-empty, it is called a random convex body. The measurability condition is then equivalent to the fact that the support function of $X$

$$
h_{X}(u)=\sup \{\langle x, u\rangle: x \in X\}, \quad u \in \mathbb{R}^{d},
$$

is a random function of $u$, where $\langle x, u\rangle$ is the scalar product. The distribution of a random convex body is uniquely identified by the finite-dimensional distributions of its support function.

A random convex closed set $X$ is said to be integrable, if there exists an integrable random vector $\xi$ such that $\xi \in X$ a.s. This vector $\xi$ is called an integrable selection of $X$. The selection expectation $\mathbf{E} X$ is the closure of the set of expectations of all its integrable selections, see (Molchanov, 2017, Sec. 2.1). The closure is not needed if

$$
\|X\|=\sup \{\|x\|: x \in X\}
$$

is an integrable random variable. Then $X$ is said to be integrably bounded.

If $X$ is integrably bounded, then $h_{X}(u)$ is integrable for all $u$, and

$$
\mathbf{E} h_{X}(u)=h_{\mathbf{E} X}(u), \quad u \in \mathbb{R}^{d} .
$$

If $X$ is integrable and $\xi$ is its integrable selection, then $h_{X}(u)=\langle\xi, u\rangle+h_{X-\xi}(u)$, whence $h_{X}(u)$ is either integrable or has the well-defined expectation $+\infty$.

If $\xi$ is a random vector in $\mathbb{R}^{d}$, then the segment $[\mathbf{0}, \xi]$ with end-points being the origin $\mathbf{0}$ and $\xi$ is a random convex body. This random convex body is integrable even for a nonintegrable $\xi$, since it contains the origin. Its expectation $Z_{\xi}=\mathbf{E}[\mathbf{0}, \xi]$ is called the zonoid of $\xi$, see (Mosler, 2002) and (Molchanov, 2017). Often, symmetrised versions of zonoids are defined as expectation of the segment $[-\xi, \xi]$ and assuming the integrability of $\xi$, see (Schneider, 2014, Sec. 3.5).

The zonoid does not uniquely determine the distribution of $\xi$, for instance, it does not change if $\xi$ is multiplied by an independent nonnegative random variable with expectation one. The extent of such nonuniqueness is explored by Molchanov et al. (2014). Despite the nonuniqueness, the zonoid delivers some information about the linear dependence between $\xi$ and $\eta$, see Dall'aglio and Scarsini $(2003)$.

It is possible to achieve the uniqueness by uplifting $\xi$ into $\mathbb{R}^{d+1}$. For this, consider the segment $[(0, \mathbf{0}),(1, \xi)]$ in $\mathbb{R}^{d+1}$, and call $\mathbf{E}[(0, \mathbf{0}),(1, \xi)]=\hat{Z}_{\xi}$ the lift zonoid of $\xi$, see (Koshevoy and Mosler, 1998) and (Mosler, 2002). Since

$$
h_{\hat{Z}_{\xi}}\left(u_{0}, u\right)=\mathbf{E}\left(u_{0}+\langle\xi, u\rangle\right)_{+}, \quad\left(u_{0}, u\right) \in \mathbb{R}^{d+1},
$$


the support function of the lift zonoid is the stop-loss transform of $\langle\xi, u\rangle$. Thus, the lift zonoid of $\xi$ determines uniquely the distribution of the scalar products $\langle\xi, u\rangle$ for all $u \in \mathbb{R}^{d}$ and so the distribution of $\xi$. This fact goes back to Hardin (1981), was independently proved by Koshevov and Mosler (1998), and further gave rise to numerous applications in multivariate analysis, see (Mosler, 2002).

This paper presents an extension of the lift zonoid concept for random convex bodies. Section 2 defines the required lifting that gives rise to the corresponding expected sets. In general, such a set is no longer a zonoid in the geometric sense of (Schneider, 2014, Sec. 3.5), and so we call it lift expectation. It is shown that the lift expectation of an integrable random convex body $X$ characterises the distributions of the support function of $X$ in any single direction $u$ such that $h_{X}(u)$ is integrable. Equivalently, the lift expectation embodies the information contained in the single marginals of a random convex function.

Examples of lift expectations are provided in Section 3 . Section 4 relates the uniqueness issue to the multivariate comonotonicity of the support function. Section [5]deals with random sets having at most a finite number of realisations. Section 6 discusses an extension of this concept for $n$-tuples of random sets. A numerical example is given in Section 7

The range of possible applications of the lift expectation is similar to those well-established for lift zonoids, see (Mosler, 2002), e.g., to assessing the depth of set-valued observations and stochastic ordering of random convex bodies. In particular, the lift expectation can be used to identify outliers in samples of random convex bodies - such samples arise in applications to partially identified problems in econometrics, see (Molchanov and Molinari, 2018). A relation to risk measures is mentioned in Example 4.1 .

\section{Lift expectation of a random set}

\subsection{Univariate distributions of the support function}

Let $X$ be a random convex body in $\mathbb{R}^{d}$. Uplift it to $\mathbb{R}^{d+1}$ by letting

$$
Y=\operatorname{conv}(\{0,0\},\{1\} \times X)
$$

be the convex hull of the origin $(0,0)$ in $\mathbb{R}^{d+1}$ and the set $\{1\} \times X$. The random convex body $Y$ is always integrable; it is integrably bounded if and only if $X$ is integrably bounded.

Definition 2.1. The set $\mathbf{E} Y$ (that is, the selection expectation of $Y$ ) is called the lift expectation of $X$ and denoted by $\hat{Z}_{X}$.

The following result establishes that the lift expectation provides exactly the same information as the distributions of the support function $h_{X}(u)$ for any given $u$ such that $h_{X}(u)$ is integrable.

Theorem 2.1. Assume that $X$ and $X^{\prime}$ are integrable random convex bodies. Then $\hat{Z}_{X}=\hat{Z}_{X^{\prime}}$ if and only if the distributions of $h_{X}(u)$ and $h_{X^{\prime}}(u)$ coincide for all $u$ such that at least one of them is integrable. 
Proof. Since the function $\left(u_{0}+t\right)_{+}$is monotonically increasing in $t$, the support function of $Y$ given by (10) is

$$
h_{Y}\left(u_{0}, u\right)=\sup \left\{\left(u_{0}+\langle x, u\rangle\right)_{+}: x \in X\right\}=\left(u_{0}+h_{X}(u)\right)_{+}
$$

for $u_{0} \in \mathbb{R}$ and $u \in \mathbb{R}^{d}$. By (Hardin, 1981, Th. 1.1), the expected value of $h_{Y}\left(u_{0}, u\right)$ considered as a function of $u_{0} \in \mathbb{R}$ uniquely determines the distribution of $h_{X}(u)$ if $h_{X}(u)$ is integrable. In the other direction, the distribution of $h_{X}(u)$ uniquely determines the expected value of $h_{Y}\left(u_{0}, u\right)$ and so $\hat{Z}_{X}$.

Remark 2.1. The lift expectation of an integrably bounded random convex body $X$ uniquely determines the one-dimensional distributions of $h_{X}(u)$ for all $u \in \mathbb{R}^{d}$. The joint distributions of the support function at different directions are not necessarily uniquely identified. The extent of the nonuniqueness for random convex bodies with the same lift expectation corresponds to the possibilities of choosing a random sublinear function on $\mathbb{R}^{d}$ with given one-dimensional marginals. Similar questions arise in the studies of stochastic processes which share onedimensional marginals with a martingale or with the Brownian motion, see Hirsch et al. (2011).

Corollary 2.2. If $X$ is integrable, then the lift expectation $\hat{Z}_{X}$ uniquely determines $\mathbf{E} X$ and $\mathbf{E} X=\{x:(1, x) \in \mathbf{E} Y\}$.

Proof. The lift expectation determines the distribution of $h_{X}(u)$ for all $u$ and so the expectation $\mathbf{E} h_{X}(u)=h_{\mathbf{E} X}(u)$. The selections of $Y$ can be obtained as $\left(\eta, \eta \xi_{\eta}\right)$, where $\eta$ is a random variable with values in $[0,1]$ and $\xi_{\eta}$ is a selection of $\eta X$. Thus, the intersection of $\mathbf{E} Y$ with the hyperplane $\left\{(1, u): u \in \mathbb{R}^{d}\right\}$ arises as the set of $\left(\mathbf{E} \eta, \mathbf{E}\left(\eta \xi_{\eta}\right)\right)$ with $\mathbf{E} \eta=1$ and so $\eta=1$ almost surely. This yields the statement, since $\xi$ is an arbitrary selection of $X$.

Remark 2.2. With each integrably bounded random convex body $X$, it is possible to associate a nested family of convex bodies $K_{n}=\mathbf{E} \operatorname{conv}\left(X_{1}, \ldots, X_{n}\right)$, $n \geq 1$, being the expectation of the convex hull of $n$ i.i.d. copies of $X$. Vitale (1987) showed that this sequence uniquely identifies the distribution of $X$ if $X$ is a singleton. Since $h_{K_{n}}(u)$ is the expectation of the maximum of $n$ i.i.d. copies of $h_{X}(u)$, the distribution of $h_{X}(u)$ for any single $u$ is uniquely identified by the sequence $\left\{h_{K_{n}}(u), n \geq 1\right\}$, see (Hoeffding, 1953). Thus, the information delivered by the nested family $\left\{K_{n}, n \geq 1\right\}$ is identical to the information recoverable from the lift expectation of $X$.

Remark 2.3. It is possible to generalise the definition of the lift expectation by replacing $Y$ with the convex hull of the origin in $\mathbb{R}^{m+d}$ and $K \times X$ for a convex body $K$ in an auxiliary space $\mathbb{R}^{m}$. Then $h_{K \times X}\left(u^{\prime}, u^{\prime \prime}\right)=\left(h_{K}\left(u^{\prime}\right)+h_{X}\left(u^{\prime \prime}\right)\right)_{+}$ for $u^{\prime} \in \mathbb{R}^{m}$ and $u^{\prime \prime} \in \mathbb{R}^{d}$. Therefore, such a generalised lift expectation still identifies only the one-dimensional marginals of $h_{X}$.

Remark 2.4. Each convex (not necessarily homogeneous) random function $\zeta(x)$, $x \in \mathbb{R}^{d}$, yields the support function of a convex body in $\mathbb{R}^{d+1}$ by letting

$$
\tilde{\zeta}(t, x)= \begin{cases}t \zeta(x / t), & t>0, \\ 0, & \text { otherwise }\end{cases}
$$


which is called the perspective transform of $f$, see (Hiriart-Urruty and Lemaréchal, 1993). Thus, the one-dimensional marginal distributions of a random convex function can be identified by a convex set in $\mathbb{R}^{d+2}$, which is the lift expectation of the random set with the support function $\tilde{\zeta}(t, x)$. The lift expectation is a convex body in $\mathbb{R}^{d+2}$, which summarises all one-dimensional marginals of a random convex function.

\subsection{Convexity for Minkowski sums and detection of outliers}

The lift expectation involves a nonlinear transformation of the support function of $X$, and so $\hat{Z}_{X+Y}$ is not necessarily equal to $\hat{Z}_{X}+\hat{Z}_{Y}$. As the following result shows, the lift expectation is a convex (with respect to the conventional set inclusion) set-valued function of integrably bounded random convex bodies.

Proposition 2.3. For integrably bounded random convex bodies $X$ and $Y$,

$$
\hat{Z}_{t X+(1-t) Y} \subset t \hat{Z}_{X}+(1-t) \hat{Z}_{Y}, \quad t \in[0,1] .
$$

Proof. It suffices to note that

$$
\begin{aligned}
& \left(u_{0}+t h_{X}(u)+(1-t) h_{Y}(u)\right)_{+} \\
& \quad \leq t\left(u_{0}+t h_{X}(u)\right)_{+}+(1-t)\left(u_{0}+t h_{Y}(u)\right)_{+} .
\end{aligned}
$$

Therefore, sections of the lift expectation

$$
\hat{Z}_{X}(\alpha)=\left\{x:(\alpha, x) \in \hat{Z}_{X}\right\}, \quad \alpha \in(0,1),
$$

are convex for Minkowski sums as function of $X$; they can be interpreted as nonlinear variants of the (linear) selection expectation. Such expectation of random variables are extensively studied, see, e.g., Peng (2004). If $Z=\{\xi\}$ is a singleton, then $\alpha^{-1} \hat{Z}_{\{\xi\}}(\alpha)$ is called the zonoid-trimmed region of $\xi$ at level $\alpha$, see (Cascos, 2010). For random vectors, such regions are used to identify outliers, also for random convex bodies they can be used to identify particularly large sets in the sample, namely those that are not contained in $\alpha^{-1} \hat{Z}_{X}(\alpha)$. The parameter $\alpha$ controls the size of this region; $\alpha$ close to one tends to regard realisations a little away from the expectation (or sample mean) as outliers. The convexity property means that if a set is not an outlier for the sample from the combination $t X+(1-t) X^{\prime}$ of two random convex bodies $X$ and $X^{\prime}$, then it arises as the convex combination of two non-outliers sampled from $X$ and $X^{\prime}$.

\subsection{Partial order generated by the lift expectation}

It is possible to partially order random convex bodies by the inclusion of their lift expectations. Theorem 2.1 yields that $\hat{Z}_{X} \subset \hat{Z}_{X^{\prime}}$ if and only if

$$
\mathbf{E}\left(u_{0}+h_{X}(u)\right)_{+} \leq \mathbf{E}\left(u_{0}+h_{X^{\prime}}(u)\right)_{+}, \quad u_{0} \in \mathbb{R},
$$

that is, for all $u \in \mathbb{R}^{d}$, the random variable $h_{X}(u)$ (if integrable) is smaller than $h_{X^{\prime}}(u)$ with respect to the increasing convex order, see (Müller and Stoyan, 2002, Th. 1.5.7). If $\mathbf{E} X=\mathbf{E} X^{\prime}$, then the expected support functions coincide and, in this case, $h_{X}(u)$ is smaller than $h_{X^{\prime}}(u)$ with respect to the convex order, see (Müller and Stovan, 2002, Th. 1.5.3). For singletons (and the corresponding lift zonoids), this is known as the lift zonoid order, see (Mosler, 2002). 


\section{Examples}

The following examples show that the lift expectation uniquely identifies the distribution of random convex bodies with restricted ranges of possible realisations. As a result, the knowledge of one-dimensional marginals might suffice to restrore the full distribution of the support function.

Example 3.1. Let $X=M+\{\xi\}$, where $M$ is a deterministic convex body and $\xi$ is an integrable random vector. Without loss of generality assume that $\mathbf{E} \xi=0$, otherwise, consider shifted $M$. Then $M=\mathbf{E} X$ is uniquely determined by $\hat{Z}_{X}$, see Corollary 2.2. Furthermore, the lift expectation determines uniquely the distribution of $h_{X}(u)=h_{M}(u)+\langle\xi, u\rangle$ for all $u$, hence, the distribution of $\xi$.

Example 3.2. Let $X=\operatorname{conv}\left\{\xi_{1}, \ldots, \xi_{n}\right\}$ be a random polytope determined by $n$ i.i.d. random copies of an integrable random vector $\xi$. The distribution of $h_{X}(u)=\max _{1 \leq i \leq n}\left\langle\xi_{i}, u\right\rangle$ yields the distribution of $\xi$ and so the distribution of $X$ is uniquely determined by its lift expectation. This is no longer the case if $\xi_{1}, \ldots, \xi_{n}$ are not i.i.d. For $d=1$, this example was considered by Cascos and Mendes (2010).

Example 3.3. Let $X$ be the sum of segments $\left[0, \xi_{i}\right], i=1, \ldots, n$, with $\xi_{1}, \ldots, \xi_{n} \in$ $\mathbb{R}_{+}^{d}$, that is, $X$ is a random zonotope in $\mathbb{R}_{+}^{d}$, and

$$
h_{X}(u)=\left\langle u, \xi_{1}\right\rangle_{+}+\cdots+\left\langle u, \xi_{n}\right\rangle_{+} .
$$

If the random vectors $\xi_{1}, \ldots, \xi_{n}$ are i.i.d., then the lift expectation identically determines the distribution of $X$. Indeed, the distribution of $h_{X}(u)$ yields the distribution of $\left\langle u, \xi_{1}\right\rangle_{+}$, and so the Laplace transform of $\xi_{1}$.

Example 3.4. Let $X=\left\{x \in \mathbb{R}^{d}:\left\langle Q^{-1} x, x\right\rangle \leq 1\right\}$ be the ellipsoid generated by a positive definite random matrix $Q$, so that $h_{X}(u)=\sqrt{\langle Q u, u\rangle}$. If $Q$ is the random diagonal matrix with positive random variables $\xi_{1}, \ldots, \xi_{d}$ on the diagonal, then the distribution of $h_{X}(u)$ yields the distribution of $\sum_{i=1} \xi_{i} u_{i}^{2}$. By taking the Laplace transform, it is immediate that the joint distribution of $\left(\xi_{1}, \ldots, \xi_{d}\right)$ (and so the distribution of $\left.X\right)$ is uniquely determined by the lift expectation of $X$. The distribution of a general (not necessarily diagonal) matrix $Q$ is not uniquely identified.

Example 3.5. Let $X=\left\{\left(\xi_{1} x_{1}, \ldots, \xi_{d} x_{d}\right):\left|x_{1}\right|+\cdots+\left|x_{d}\right| \leq 1\right\}$ be the $\ell_{1}$-ball scaled by the components of $\xi=\left(\xi_{1}, \ldots, \xi_{d}\right) \in(0, \infty)^{d}$. Then

$$
h_{X}(u)=\max _{i=1, \ldots, d}\left|\xi_{i} u_{i}\right|
$$

and its distributions for each $u \in(0, \infty)^{d}$ uniquely determine the distribution of $\xi$, since

$$
\mathbf{P}\left\{h_{X}(u) \leq t\right\}=\mathbf{P}\left\{\xi_{1} \leq t u_{1}^{-1}, \ldots, \xi_{d} \leq t u_{d}^{-1}\right\} .
$$

Thus, the lift expectation of $X$ uniquely determines the distribution of $\xi$ and so that of $X$. The (nonlifted) expectation of the so defined $X$ arises in the theory of extreme values; it is called a max-zonoid, see (Molchanov, 2008). 


\section{Uniqueness of distribution and comonotonicity}

In the one-dimensional case, $X=[\xi, \eta]$ is a random segment determined by two integrable random variables $\xi$ and $\eta$ coupled so that $\mathbf{P}\{\xi \leq \eta\}=1$.

Example 4.1. If $X=[\xi, \eta]$, then $\hat{Z}_{X}$ is a subset of the half-plane $[0, \infty) \times \mathbb{R}$ with

$$
h_{\hat{Z}_{X}}\left(u_{0}, u\right)=\left\{\begin{array}{ll}
\mathbf{E}\left(u_{0}+u \eta\right)_{+} & u \geq 0, \\
\mathbf{E}\left(u_{0}+u \xi\right)_{+} & u<0,
\end{array} \quad\left(u_{0}, u\right) \in \mathbb{R}^{2} .\right.
$$

An example of the set $\hat{Z}_{X}$ is shown on Figure 1. The upper bound of $\hat{Z}_{X}$ is the upper Lorenz curve of $\eta$, while the lower bound of $\hat{Z}_{X}$ is the lower Lorenz curve of $\xi$. The scaled vertical sections of $\hat{Z}_{X}$ are intervals given by

$$
\alpha^{-1}\left\{x:(\alpha, x) \in \hat{Z}_{X}\right\}=[\inf \mathbf{E}(\zeta \xi), \sup \mathbf{E}(\zeta \eta)], \quad \alpha \in(0,1],
$$

where the infimum and supremum are taken over random variables $\zeta \in\left[0, \alpha^{-1}\right]$ such that $\mathbf{E} \zeta=1$. This interval equals $\left[-\operatorname{AVaR}_{\alpha}(\xi), \operatorname{AVaR}_{\alpha}(-\eta)\right]$, where $\operatorname{AVaR}_{\alpha}$ denotes the Average Value-at-Risk at level $\alpha$, see, e.g., (Föllmer and Schied, 2004, Def. 4.43).

The lift expectation of $X=[\xi, \eta]$ yields only the marginal distributions of $\xi$ and $\eta$, and the uniqueness issue consists in the existence of a unique measure on the half-plane $H=\left\{\left(x_{1}, x_{2}\right): x_{1} \leq x_{2}\right\}$ with given marginals. The joint distribution of $\xi$ and $\eta$ is unique if it is known that the random vector $(\xi, \eta)$ is supported by a set $A \subset H$ such that $A$ intersected with each horizontal or vertical line is a singleton, equivalently, if one knows the copula of $(\xi, \eta)$. The following result characterises the uniqueness cases in relation to the comonotonicity properties of the end-points.

Proposition 4.1. The distribution of $X=[\xi, \eta]$ is uniquely determined by its lift expectation if the end-points $\xi$ and $\eta$ are comonotonic, that is, $\xi=f_{1}(\zeta)$ and $\eta=f_{2}(\zeta)$ for two monotone functions $f_{1}$ and $f_{2}$ and a random variable $\zeta$.

Proof. By Theorem 2.1 the lift expectation of $X$ uniquely determines the marginal distributions of random vector $(\xi, \eta)$, and so its joint distribution in view of comonotonicity, see (Puccetti and Scarsini, 2010).

The same result holds if $(-\xi, \eta)$ is comonotonic. Without the comonotonicity assumption, the result does not hold, e.g., $X$ taking values $[1,3]$ or $[2,4]$ with equal probabilities and $X^{\prime}$ taking values $[1,4]$ and $[2,3]$ with equal probabilities share the same one-dimensional distributions of the support function. However, if the probabilities of these two values of $X$ are different, then the distribution of $X$ is uniquely identified, see Proposition 5.1 . The next result follows from the uniqueness of strongly comonotonic vectors with given marginal distributions, see (Puccetti and Scarsini, 2010, Rem. 3.4). 
Proposition 4.2. The distribution of an integrably bounded random convex compact set $X$ in $\mathbb{R}^{d}$ is uniquely determined by its lift expectation if there exists a deterministic function $g: \mathbb{S}^{d-1} \mapsto\{-1,1\}$ such that $g\left(u_{1}\right) h_{X}\left(u_{1}\right), \ldots, g\left(u_{n}\right) h_{X}\left(u_{n}\right)$ form a comonotonic vector for all $u_{1}, \ldots, u_{n} \in \mathbb{S}^{d-1}$ and $n \geq 2$.

In particular, Proposition 4.2 applies if $h_{X}(u)=f(u, \eta)$, where $\eta$ is a random variable and the function $f(u, \cdot)$ is monotone for each $u \in \mathbb{S}^{d-1}$.

If $h_{X}(u)$ is distributed as a random variable $\zeta$ for all $u$ with $\|u\|=1$ (e.g., if $X$ is isotropic), then the lift expectation of $X$ has the support function $\mathbf{E}\left(u_{0}+\right.$ $\zeta\|u\|)_{+}$. The lift expectation of $X$ determines the distribution of $\zeta$, but not the distribution of $X$, for instance, $X$ shares the lift expectation with the centred ball of radius $\zeta$.

Example 4.2. Let $X$ be an isotropic rotation of a deterministic origin symmetric convex body $K$. Then $h_{X}(u)$ has the same distribution for all $u$ and $\mathbf{P}\left\{h_{X}(u) \leq t\right\}$ for $\|u\|=1$ equals the $(d-1)$-dimensional Hausdorff measure (normalised by the area of the unit sphere) of the set $\left\{u \in \mathbb{S}^{d-1}: h_{K}(u) \leq t\right\}$. Then

$$
\left\{u \in \mathbb{S}^{d-1}: h_{K}(u) \leq t\right\}=t\left\{v:\|v\|=t^{-1}, h_{K}(v) \leq 1\right\}=\left(t \mathbb{S}^{d-1}\right) \cap K^{o},
$$

where $K^{o}=\left\{v: h_{K}(v) \leq 1\right\}$ is the polar body to $K$. Equivalently, the onedimensional distributions of $h_{X}$ can be retrieved from the Lebesgue measure of $B_{r} \cap K^{o}$ for all $r>0$. These values do not suffice to retrieve (up to a rotation) a general convex body $K$.

\section{Discrete distributions}

Assume that $X$ is a random convex body that takes a finite number of values $K_{1}, \ldots, K_{N}$ with $\mathbf{P}\left\{X=K_{i}\right\}=p_{i}, i=1, \ldots, N$.

Proposition 5.1. If $X$ takes a finite number of possible values and their probabilities $p_{1}, \ldots, p_{N}$ are all different, then the distribution of $X$ is uniquely determined by its lift expectation.

Proof. Let $\left\{u_{n}, n \geq 1\right\}$ be a countable dense set on $\mathbb{S}^{d-1}$. The lift expectation yields the possible values $t_{n 1}, \ldots, t_{n m_{n}}$ of $h_{X}\left(u_{n}\right)$, where $m_{n} \leq N$. Since there is a $u_{n}$ such that $h_{K_{i}}\left(u_{n}\right), i=1, \ldots, N$, are all different, we have $\sup _{n \geq 1} m_{n}=N$, and so it is possible to recover $N$. The supremum is attained, and so one obtains the probabilities $p_{1}, \ldots, p_{N}$ and the values $h_{K_{1}}\left(u_{n}\right), \ldots, h_{K_{N}}\left(u_{n}\right)$ for some $u_{n}$. The values $h_{K_{i}}(u)$ can be retrieved by tracing the distribution of $h_{X}(u)$ over $u$ from the unit sphere.

If the number of realisations is countable, then it is not possible to recover all individual support functions in some direction in order to subsequently trace them as in the proof of Proposition 5.1. If the probabilities are not different, then the uniqueness fails, as the discussion following Proposition 4.1] shows. However, 
the non-equal probabilities ensure the uniqueness even if the comonotonicity condition fails.

The support set of a convex body $K$ is defined by

$$
H_{K}(u)=\left\{x \in K:\langle u, x\rangle=h_{K}(u)\right\}, \quad u \neq 0 .
$$

The support set is a singleton, if $K$ is strictly convex, that is, its boundary does not contain any nontrivial segment. The support function of a strictly convex set is differentiable and its gradient $h_{K}^{\prime}(u)$ equals the support point $H_{K}(u)$ if $\|u\|=1$, see (Schneider, 2014, Cor. 1.7.3).

Theorem 5.2. Assume that the random convex body $X$ has realisations $K_{1}, \ldots, K_{N}$, which are strictly convex and such that $H_{K_{i}}(u) \neq H_{K_{j}}(u)$ for all $i \neq j$ and all $u \neq 0$. Then the lift expectation of $X$ uniquely determines its possible realisations and their probabilities.

Proof. There is a $u \in \mathbb{S}^{d-1}$ such that $h_{X}(u)$ takes $N$ different possible values, and this $u$ can be identified from the one-dimensional distributions of the support function as the point where the number of different values is the largest. Assume that these values are $s_{1}, \ldots, s_{n}$ with probabilities $p_{1}, \ldots, p_{n}$, and note that some or all of the probabilities may be equal.

Given this $u$, we assign the determined values to the support function, so let $h_{K_{i}}(u)=s_{i}, i=1, \ldots, n$. In a neighbourhood of $u$, the values of the support functions are still different, and it is possible to recover their probabilities there. We let this neighbourhood $U$ grow until, at some point $v \in \partial U, h_{K_{i}}(v)=h_{K_{j}}(v)$ for some $i \neq j$. This equality is only possible at an isolated point, since otherwise the gradients of support functions of $K_{i}$ and $K_{j}$ would be equal and these two sets would have identical support points.

The knowledge of the support functions of $K_{i}$ and $K_{j}$ on $U$ makes it possible to calculate the gradients at $v$ (which are different for all $i$ by the assumption) and so identify the support functions of $K_{i}$ and $K_{j}$ in a neighbourhood of $v$. Continuing this, it is possible to completely identify the support functions of all possible realisations.

\section{Tuples of random convex bodies}

Let $\left(X_{1}, \ldots, X_{n}\right)$ be an $n$-tuple of integrably bounded random convex bodies in $\mathbb{R}^{d}$. Their lift expectation is a convex body in $\mathbb{R}^{n d+1}$ being the expectation of the convex hull of the origin and the set $\{1\} \times X_{1} \times \cdots \times X_{n}$. By Theorem 2.1 . this lift expectation uniquely determines the distribution of

$$
h_{X_{1}}\left(u^{(1)}\right)+\cdots+h_{X_{n}}\left(u^{(n)}\right), \quad u^{(1)}, \ldots, u^{(n)} \in \mathbb{R}^{d} .
$$

The following result addresses the case when $X_{1}=\cdots=X_{n}=X$. Ordering of such lift expectations by inclusion corresponds to the ordering of $n$ dimensional distributions of $h_{X}$ in the increasing positive linear convex order, see (Müller and Stoyan, 2002, Def. 3.5.1). 
Theorem 6.1. Let $X$ be an integrably bounded random convex body which almost surely contains the origin. The sequence of lift expectations of $(X, \ldots, X)$ for $n$-tuples of the same $X$ and all $n \geq 1$ uniquely determines the distribution of $X$.

Proof. The lift expectation of the $n$-tuple $(X, \ldots, X)$ determines the distribution of $h_{X}\left(u^{(1)}\right)+\cdots+h_{X}\left(u^{(n)}\right)$. By replacing $u^{(i)}$ with $t_{i} u^{(i)}$ with $t_{i} \geq 0$, we see that the distribution of the scalar product of $\left(h_{X}\left(u^{(1)}\right), \ldots, h_{X}\left(u^{(n)}\right)\right)$ and $\left(t_{1}, \ldots, t_{d}\right)$ is uniquely determined. Since the values of the support function are nonnegative, the Laplace transform and so the joint distribution of $\left(h_{X}\left(u^{(1)}\right), \ldots, h_{X}\left(u^{(n)}\right)\right)$ is uniquely determined.

Example 6.1. The distribution of $X=[\xi, \eta]$ is uniquely determined by the expectation of the convex hull of the origin in $\mathbb{R}^{3}$ and $\{1\} \times[\xi, \eta] \times[\xi, \eta]$. The projections of this expected set on the first two coordinates or the first and the third coordinates determine the marginal of $\xi$ and $\eta$, while the projection on the last two coordinates is the zonoid of $(\xi, \eta)$. Note that the lift expectation differs from the lift zonoid of $(\xi, \eta)$.

\section{Numerical example}

Let $X=[\xi, \eta]$ be a segment on the positive half-line. In econometrics such segments appear as the data on salary brackets, given by respondents reluctant to report the exact value of their salaries. The lift expectation of $X$ is then a convex set in the plane. The lower boundary of this set coincides with the lower boundary of the lift zonoid of $\xi$ (also called the generalised lower Lorenz curve of $\xi$ ) and the upper boundary stems from the lift zonoid of $\eta$ (the generalised upper Lorenz curve of $\eta$ ).

We illustrate the above argument with the US Current Population Survey 2016 freely available from the US Census website. The variable of interest to us is the personal income variable denoted by ptot_ $r$ in the dataset. This income variable takes values from the set $\{1,2, \ldots, 41\}$. Its value $i \in\{1, \ldots, 40\}$, means that the personal income of the observed individual lies in the interval $\left[a_{i}, b_{i}\right]$, where $b_{i}-a_{i}=2499$, and $a_{i}=b_{i-1}+1$, with $a_{1}=0$. We removed from the dataset the individuals with the value ptot $r=41$, meaning that the observed individual earns at least $100000 \$$, and so the corresponding interval is unbounded. Our final sample includes 132,410 observations.

Figure 1 shows the estimate for the lift expectation calculated by taking the mean value of the sets $\{1\} \times\left[a_{i}, b_{i}\right]$ on the plane. The lower bound (solid black curve) is the Lorenz curve for the lower bounds of the observed intervals; the upper bound (red dashed curve) is the upper Lorenz curve for the upper bounds. The (blue thick) vertical segment with the the $x$-coordinate 1 is the mean $[27204.40,29450.42]$, obtained by averaging the lower and upper bounds, see Corollary 2.2

The area of a lift zonoid of a random variable is the Gini mean difference, which can be used as an inequality index, see, e.g., (Mosler, 2002). In view 


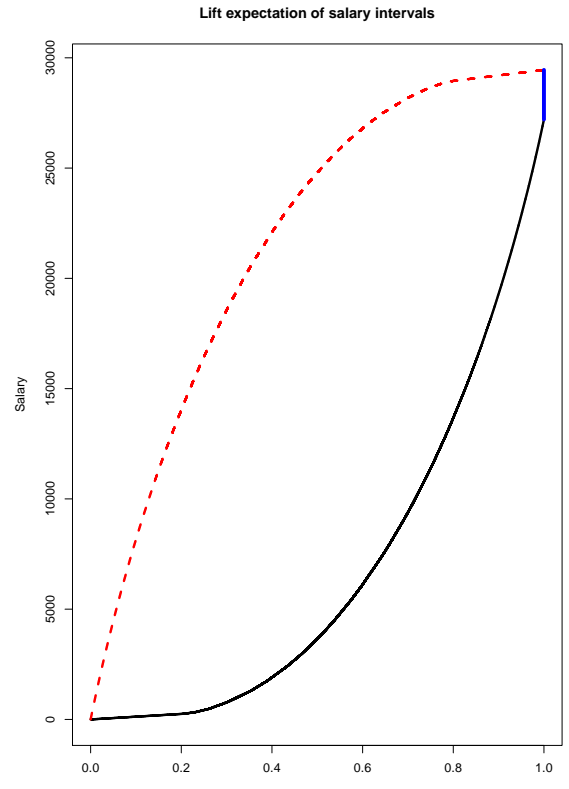

Figure 1: Estimate of the lift expectation for the interval salary data from CPS 2016.

of this, the area of the lift expectation provides an upper bound for the Gini inequality index of any random variable lying in $X=[\xi, \eta]$. In view of this, the area of $\hat{Z}_{X}$ may be used as an inequality measure for the case of interval-valued responses.

\section{Acknowledgement}

The authors are grateful to the Labex MME-DII and the University ParisSaclay (EPEE, Univ-Evry) for hosting a conference where they all met and initiated this project. The authors are grateful to Ignacio Cascos for helpful comments and the idea of Example 6.1. GK was supported by the Russian Science Foundation grant RSF 14-50-00150. IM was supported by the Swiss National Foundation grants IZ73Z0_152292 and 200021_153597.

The authors are grateful to the two anonymous referees and the associated editor whose comments have led to substantial improvements of the paper.

\section{References}

Cascos, I., 2010. Data depth: multivariate statistics and geometry. In: Kendall, W. S., Molchanov, I. (Eds.), New Perspectives in Stochastic Geometry. Oxford University Press, Oxford, pp. 398-426. 
Cascos, I., Mendes, M., 2010. Lorenz curves of extrema. In: Borgelt, C. et al. (Eds.), Combining Soft Computing and Statistical Methods in Data Analysis. Springer, Berlin, pp. 81-88.

Dall'aglio, M., Scarsini, M., 2003. Zonoids, linear dependence, and size-biased distributions on the simplex. Adv. Appl. Probab. 35, 871-884.

Föllmer, H., Schied, A., 2004. Stochastic Finance. An Introduction in Discrete Time, 2nd Edition. De Gruyter, Berlin.

Hardin, C. D. J., 1981. Isometries on subspaces of $L^{p}$. Indiana Univ. Math. J. $30,449-465$.

Hiriart-Urruty, J.-B., Lemaréchal, C., 1993. Convex Analysis and Minimization Algorithms. Vol. 1 and 2. Springer, Berlin.

Hirsch, F., Profeta, C., Roynette, B., Yor, M., 2011. Peacocks and Associated Martingales, with Explicit Constructions. Springer, Milan; Bocconi University Press, Milan.

Hoeffding, W., 1953. On the distribution of the expected values of the order statistics. Ann. Math. Statist. 24, 93-100.

Koshevoy, G. A., Mosler, K., 1998. Lift zonoids, random convex hulls and the variability of random vectors. Bernoulli 4, 377-399.

Molchanov, I., 2008. Convex geometry of max-stable distributions. Extremes $11,235-259$.

Molchanov, I., 2017. Theory of Random Sets, 2nd Edition. Springer, London.

Molchanov, I., Molinari, F., 2018. Random Sets in Econometrics. Econometric Society Monographs. Cambridge Univ. Press, Cambridge.

Molchanov, I., Schmutz, M., Stucki, K., 2014. Invariance properties of random vectors and stochastic processes based on the zonoid concept. Bernoulli 20, $1210-1233$.

Mosler, K., 2002. Multivariate Dispersion, Central Regions and Depth. The Lift Zonoid Approach. Vol. 165 of Lect. Notes Statist. Springer, Berlin.

Müller, A., Stoyan, D., 2002. Comparison Methods for Stochastic Models and Risks. Wiley, Chichester.

Peng, S., 2004. Nonlinear expectations, nonlinear evaluations and risk measures. In: Stochastic Methods in Finance. Vol. 1856 of Lecture Notes in Math. Springer, Berlin, pp. 165-253.

Puccetti, G., Scarsini, M., 2010. Multivariate comonotonicity. J. Multivariate Anal. 101, 291-304. 
Schneider, R., 2014. Convex Bodies. The Brunn-Minkowski Theory, 2nd Edition. Cambridge University Press, Cambridge.

Vitale, R. A., 1987. Expected convex hulls, order statistics, and Banach space probabilities. Acta Appl. Math. 9, 97-102. 